\begin{tabular}{|c|c|}
\hline Title & Effects of drug discontinuation after short-term daily al endronate administration on osteoblasts and osteocytes in mice \\
\hline Author(s) & $\begin{array}{l}\text { T suboi, Kanako; Hasegawa, Tomoka; Y amamoto, Tomomaya; Sasaki, Muneteru; Hongo, Hiromi; Luiz de Freitas, } \\
\text { Paulo Henrique; Shimizu, Tomohiro; T akahata, Masahiko; Oda, Kimimitsu; Michigami, T oshimi; Li, Minqi; Kitagawa, } \\
\text { Y oshimasa; A mizuka, Norio }\end{array}$ \\
\hline Citation & $\begin{array}{l}\text { Histochemistry and cell biology, 146(3), 337-350 } \\
\text { https://doi.org/10.1007/s00418-016-1450-7 }\end{array}$ \\
\hline Issue Date & 2016-09 \\
\hline Doc URL & http:/hdl.handle.net/2115/67072 \\
\hline Rights & The final publication is available at Springer via http://dx.doi.org/10.1007/s00418-016-1450-7 \\
\hline Type & article (author version) \\
\hline File Information & Histochem. Cell Biol.146(3).pdf \\
\hline
\end{tabular}

Instructions for use 


\title{
Effects of drug discontinuation after short-term daily alendronate administration on osteoblasts and osteocytes in mice
}

\author{
Kanako Tsuboi $^{1,2}$, Tomoka Hasegawa ${ }^{1}$, Tomomaya Yamamoto ${ }^{1}$, Muneteru Sasaki ${ }^{3}$, \\ Hiromi Hongo ${ }^{1}$, Paulo Henrique Luiz de Freitas ${ }^{4}$, Tomohiro Shimizu ${ }^{5}$, Masahiko \\ Takahata $^{5}$, Kimimitsu Oda ${ }^{6}$, Toshimi Michigami ${ }^{7},{\text { Minqi } \text { Li }^{8} \text {, Yoshimasa Kitagawa }}^{2}$ and \\ Norio Amizuka ${ }^{1}$
}

Departments of ${ }^{1}$ Developmental Biology of Hard Tissue, ${ }^{2}$ Oral Diagnosis and Medicine, Graduate School of Dental Medicine, Hokkaido University, Sapporo, Japan, ${ }^{3}$ Department of Applied Prosthodontics, Unit of Translational Medicine, Graduate School of Biomedical Sciences, Nagasaki University, Nagasaki, Japan, ${ }^{4}$ Department of Dentistry, Federal University of Sergipe at Lagarto, Brazil, ${ }^{5}$ Department of Orthopedic Surgery, Graduate School of Medicine, Hokkaido University, Sapporo, Japan, ${ }^{6}$ Division of Biochemistry, Niigata University Graduate School of Medical and Dental Sciences, Niigata, Japan, ${ }^{7}$ Department of Bone and Mineral, Research Institute, Osaka Medical Center for Maternal and Child Health, Osaka, Japan, ${ }^{8}$ Shandong Provincial Key Laboratory of Oral Biomedicine, The School of Stomatology, Shandong University, Jinan, China

\section{Running title: Effects of ALN on osteoblasts and osteocytes}

Keywords: bisphosphonates, osteocyte, discontinuation, osteoblast

\section{Address for correspondence:}

\author{
Norio Amizuka, DDS, PhD \\ Department of Developmental Biology of Hard Tissue \\ Graduate School of Dental Medicine, Hokkaido University \\ Kita 13 Nishi 7 Kita-ku \\ Sapporo, 060-8586, Japan \\ Tel: +81-11-706-4223 \\ Fax: +81-11-706-4226 \\ E-mail: amizuka@den.hokudai.ac.jp
}




\begin{abstract}
In order to determine whether osteoclastic bone resorption is restarted after withdrawn of bisphosphonates, we conducted histological examinations on murine osteoclasts, osteoblasts and osteocytes after discontinuation of a daily regimen of alendronate (ALN) with a dosage of $1 \mathrm{mg} / \mathrm{kg} /$ day for 10 days. After drug discontinuation, metaphyseal trabecular number and bone volume remained unaltered for the first 4 days. Osteoclast number did not increase, while the number of apoptotic osteoclasts was elevated. On the other hand, tissue non-specific alkaline phosphatase (ALPase)-immunoreactive area was markedly reduced after ALN discontinuation. In addition, osteocytes showed an atrophic profile with empty lacunar areas during and after ALN treatment. Interestingly, as early as 36 hrs after a single ALN injection, osteocytes show signs of atrophy despite the presence of active osteoblasts. Structured illumination microscopy system showed shortening of osteocytic cytoplasmic processes after drug cessation, suggesting a possible morphological and functional disconnection between osteocytes and osteoblasts. Taken together, it appears that osteoclastic bone resorption is not resumed after ALN discontinuation; also, osteoblasts and osteocytes hardly seem to recover once they are inactivated and atrophied by ALN. In summary, it seems that one must pay more attention to the responses of osteoblasts and osteocytes, rather focusing on the resuming of osteoclastic bone resorption after the ALN discontinuation.
\end{abstract}




\section{Introduction}

Alendronate (ALN) is the most commonly used nitrogen-containing bisphosphonate for osteoporotic treatment. In general, nitrogen-containing bisphosphonates - pamidronate, alendronate, ibandronate, zoledronate, and risedronate - inhibit farnesyl pyrophosphate synthase, an important enzyme in the mevalonate pathway [1, 2]. Inhibition of the mevalonate pathway results in impaired protein prenylation, especially that of the small GTPase of the Ras family [3]. Small GTPase proteins are important for vesicular trafficking and cell survival, and they are involved in cytoskeletal organization of bone-resorbing osteoclasts [4, 5]. Indeed, Väänänen's group has demonstrated that nitrogen-containing bisphosphonates keep osteoclasts from resorbing bone mostly due to disrupted vesicular trafficking and cytoskeleton misassemble [6]. ALN has a high affinity for crystalline calcium phosphates, and therefore, accumulates on bone surfaces being resorbed by osteoclasts, which incorporate the drug almost immediately [7]. Thus, osteoclasts seem to be the primary target for ALN.

However, bone formation is coupled with bone resorption during the process of physiological remodeling, which continuously takes place along the bone surfaces with resorption preceding formation. There is evidence supporting the hypothesis that osteoclastic bone resorption would trigger the differentiation and activation of osteoblasts, a process referred to as a “coupling phenomenon” [8-11]. Without osteoclasts, osteoblastic population, osteoblastic bone formation and bone mineralization are markedly diminished in op/op mice, which lack the macrophage colony-stimulating factor [12, 13]. One of our studies demonstrated that cell coupling between osteoclasts and preosteoblasts must take place for the parathyroid hormone-driven bone anabolic effect to occur [14]. Therefore, the drop in osteoclastic bone resorption induced by bisphosphonate administration may cause a reduction both in osteoblastic population and in bone formation. Such assumption is based on reports showing that bisphosphonates inhibited bone formation as measured by mineral apposition rate in rats [15, 16], mice [17] and dogs [18].

We assume another possibility, which is that inhibition of bone formation and osteoblast inactivation might indirectly affect osteocytes embedded in bone. All osteocytes lie within osteocytic lacunae and connect to other osteocytes and to osteoblasts through thin cytoplasmic processes that pass through narrow osteocytic canaliculi [19-21]. Furthermore, osteoblasts 
and osteocytes connect their cytoplasmic processes by means of gap junctions [22-24], thereby forming a functional syncytium. Thus, it is plausible that significant inhibition of osteoclast and osteoclastic bone resorption would affect the biological activities of not only osteoblasts but also that of osteocytes.

Some issues may arise when bisphosphonates are suddenly withdrawn as preventive measure for avoiding bisphosphonate-related osteonecrosis of the jaws (BRONJ), for example [25-27]. Since ALN primarily targets bone-resorbing osteoclasts and accumulates on the bone surface, the drug may not affect osteoclast precursors. One may wonder if osteoclasts derived from those supposedly non-affected precursors start resorbing bone upon bisphosphonate discontinuation, and also what happens to osteoblasts and osteocytes once the drug is discontinued. In an attempt to shed a light on these issues, we have examined the behavior of osteoclasts, osteoblasts and osteocytes in mice at 12 hrs, 1, 2, 3 and 4 days after discontinuation of a daily ALN regimen (1 mg/kg for 10 days). 


\section{Materials and methods}

\section{Animals and tissue preparation for histochemistry and electron microscopy}

All animal experiments in this study were approved and conducted under the Hokkaido University Guidelines for Animal Experimentation. Ninety-six 6 weeks-old female ICR mice (CLEA Japan, Inc., Tokyo, Japan) were received daily subcutaneous injections of $1 \mathrm{mg} / \mathrm{kg}$ of ALN for 10 days. Some mice were fixed at 5 days after the first injection and others were fixed immediately ( 0 hr), 12 hrs, 1, 2, 3 and 4 days after the last injection $(\mathrm{n}=12$ for each) (Fig. 1). Mice without ALN administration was used for the control experiments. Forty eight mice ( $\mathrm{n}=6$ for each group) were perfused with $4 \%$ paraformaldehyde in a $0.1 \mathrm{M}$ cacodylate buffer ( $\mathrm{pH}$ 7.4) through the left ventricle under anesthesia with diethylether inhalation followed by an intraperitoneal injection of chloral hydrate. Femora and tibiae were removed en bloc and immediately immersed in the same fixative for $18 \mathrm{hrs}$. Left femora and tibiae were used for histochemical analyses, while right ones were employed for examination by microCT (femora) and transmission electron microscopy (TEM, tibiae). For examining the results of a single injection of alendronate, thirty-six 6 weeks-old female ICR mice were injected with alendronate $(1 \mathrm{mg} / \mathrm{kg})$ at the external jugular vein and then fixed after 3, 9, 24, 36 and 48 hrs ( $\mathrm{n}=6$ for each) as described above. Specimens were decalcified with 10\% EDTA for 2 months prior to paraffin and OCT compound embedding. Paraffin sections were cut sagittally and parallel to the longitudinal axis of the bone prior to histochemistry. All sections were photographed with a Nikon Eclipse E800 microscope (Nikon Instruments Inc. Tokyo, Japan) and a digital camera (Nikon DXM1200C, Nikon). OCT compound sections were sliced into $20 \mu \mathrm{m}$-thick with a cryostat (Jung Research Cryostat Model CM3000, Leica Microsystems, GmbH, Wetzlar, Germany) for rhodamine-phalloidine staining.

For TEM observation, the extracted right tibiae were immediately immersed in a solution containing 2\% paraformaldehyde and $2.5 \%$ glutaraldehyde diluted in a $0.067 \mathrm{M}$ cacodylate buffer ( $\mathrm{pH}$ 7.4) for 18 hrs. TEM specimens were decalcified with 5\% EDTA for 2 months. They were then post-fixed in a mixture of $1 \%$ osmium tetraoxide with $1.5 \%$ potassium ferrocyanide in $0.1 \mathrm{M}$ a cacodylate buffer ( $\mathrm{pH} 7.4$ ) for 4 hrs, dehydrated with ascending concentrations of acetone, and then embedded in epoxy resin (Taab, Berkshire, UK). 120nm-thick ultrathin sections were obtained with an ultramicrotome (Sorvall MT-5000; Ivan Sorvall. Inc., Norwalk, CT). Sections were stained with uranyl acetate and lead citrate prior 
to examination under a TEM (Hitachi H-7100 Hitachi Co. Ltd, Tokyo, Japan) at $80 \mathrm{kV}$.

\section{MicroCT analysis}

Femora of mice fixed at 5 days after the first injection, and others fixed immediately $(0 \mathrm{hr})$, 12 hrs, 1, 2, 3 and 4 days after the last injection ( $n=6$ for each group) were used for microCT analysis (R_mCT2; Rigaku, Tokyo, Japan, tube voltage $60 \mathrm{kV}$ ) obtained at an isotropic resolution of $10-\mu \mathrm{m}$. The regions of interest (ROI) were defined as the distal femoral metaphyses and a diaphyseal area $7 \mathrm{~mm}$ away from the articular surface, both including the cortical plates.

\section{Histochemical staining of ALPase and TRAPase}

Dewaxed paraffin sections were examined for tissue non-specific alkaline phosphatase (ALPase) as previously reported [28, 29]. In brief, sections were immersed in methanol containing $0.3 \% \mathrm{H}_{2} \mathrm{O}_{2}$ for 30 mins to block endogenous peroxidases. To reduce non-specific binding, 1\% bovine serum albumin (Seologicals Proteins Inc. Kankakee, IL) in PBS (1\%BSA-PBS) was applied to the sections for 20 mins. It was followed by incubation with rabbit polyclonal antiserum against ALPase [30] at a dilution of 1: 200 with 1\%BSA-PBS at room temperature (RT) for 2 hrs. Following several washings in PBS, sections were incubated with horseradish peroxidase (HRP)-conjugated anti-rabbit IgG (Chemicon International Inc., Temecula, CA) for 1 hr. Diaminobenzidine tetrahydrochloride was used to visualize the immunoreaction. For tartrate-resistant acid phosphatase (TRAPase) visualization [31], sections were incubated in a mixture of $8 \mathrm{mg}$ of naphthol AS-BI phosphate (Sigma, St. Louis, MO), $70 \mathrm{mg}$ of red violet LB salt (Sigma) and $50 \mathrm{mM}$ $\mathrm{L}(+)$ tartaric acid (0.76g, Nacalai Tesque, Kyoto, Japan) diluted in $0.1 \mathrm{M}$ sodium acetate buffer ( $\mathrm{pH} \mathrm{5.0)}$ ) for 20 mins at $37^{\circ} \mathrm{C}$. For double ALPase-TRAPase detection, ALPase immunostaining was performed prior to TRAPase histochemistry as described above.

\section{TUNEL staining}

TUNEL, TdT mediated dUTP nick end labeling, was performed according to the manufacturer's instructions (Trevigen Inc., Gaithersburg, MD). In brief, dewaxed paraffin sections were treated with proteinase $\mathrm{K}$ for $15 \mathrm{~min}$ at RT, and then, were washed with PBS. The sections were immersed in quenching solution for $5 \mathrm{~min}$, subsequently incubated with $1 \mathrm{X}$ TdT labeling buffer for $5 \mathrm{~min}$, reacted with labeling reaction mix for $60 \mathrm{~min}$ at $37^{\circ} \mathrm{C}$, and 
were incubated in $1 \mathrm{X}$ TdT stop buffer for 5 min. They were treated in strep-HRP solution for $10 \mathrm{~min}$ at $37^{\circ} \mathrm{C}$. For double detection of TRAPase and TUNEL, TUNEL staining was performed prior to TRAPase enzyme histochemistry.

\section{SIM and confocal laser scanning microscopic observation for actin filaments and chondroitin-4-sulfate}

For detection of chondroitin-4-sulfate, chondroitinase ABC (Sigma, St. Louis, MO) diluted in $100 \mathrm{mM}$ Tris acetate buffer $(0.2 \mathrm{U} / \mathrm{ml})$ was applied on the sections at $37^{\circ} \mathrm{C}$ for $1 \mathrm{hr}$. The sections were subsequently incubated with anti-chondroitin-4-sulfate monoclonal antibody 2B6 (Cosmo Bio, Co., Ltd., Tokyo, Japan) at a dilution of 1: 20 for 3 hrs at RT, and then incubated with FITC-conjugated anti-mouse IgGs (MP Biomedicals, LLC., Santa Ana, CA) at a dilution of 1:100 for $1 \mathrm{hr}$ at RT. The sections were observed under confocal laser scaning microscopy (FV1000, Olympus, Co., Tokyo, Japan). Frozen section with 20 $\mu$ m thickness were incubated in a solution of rhodamine-phalloidine (Molecular Probes. Inc., Eugene, OR) for 3 days. The images were acquired by a structured illumination microscopy system (SIM) (N-SIM, Nikon Instruments, Nikon Imaging Center at Hokkaido University) equipped with an Eclipse Ti-E inverted microscope using CFI Apo TIRF x 100 oil objective lens, and were processed with the NIS-Elements software (Nikon Instruments).

Histomorphometry or BV/TV, Tb.N, Tb.Th and Tb.Sp, the quantification of ALPase-positive area and the number of TRAPase-reactive osteoclasts, and the index of TRAPase/TUNEL-double positive osteoclasts

A $400 \mu \mathrm{m} \times 600 \mu \mathrm{m}$ assumed boxed area (ROI; region of interest) of central metaphyseal region, $150 \mu \mathrm{m}$ below the growth plate of the femoral metaphysis was employed for assessment of the following static parameters of bone histomorphometry: bone volume/tissue volume (BV/TV), trabecular number (Tb.N), trabecular thickness (Tb.Th), trabecular separation (Tb.Sp). The number of TRAPase-reactive osteoclasts and the percentage of TRAPase/TUNEL-double positive cells (osteoclasts) were also examined in the same ROI of all the experimental groups. The index of TRAPase/TUNEL-double positive osteoclasts was represented as a percentage by dividing the total numbers of TRAPase-reactive osteoclasts.

For quantifying the area occupied by ALPase-positive osteoblasts in the secondary trabecular regions of the control and experimental specimens, images of sections stained for ALPase in an assumed box - 400 $\mu \mathrm{m}$ x $400 \mu \mathrm{m}$ square at the central metaphyseal region, 800 $\mu \mathrm{m}$ below the growth plate, excluding the cortical bone - were obtained. 
ALPase-immunopositive areas were quantified using the ImagePro Plus 6.2 software (Media Cybernetics, Silver Spring, MD), and the index was expressed as a percentage of bone volume.

\section{Quantification of atrophic osteocytes and the numbers of cytoplasmic processes extended} from an individual osteocyte

With regard to atrophic osteocytes, we divided osteocytes into intact and atrophic cells, the latter being categorized as a shrunken cell (less than two-thirds its lacunar area) with an irregular shape not matching the configuration of its lacunae. The index of the atrophic osteocytes was expressed as the percentage of the atrophic cells divided with the total numbers of osteocytes. Under SIM observation of rhodamine-phalloidine staining, the numbers of cytoplasmic processes per an osteocyte were counted to estimate the average numbers of cytoplasmic processes extended from an individual osteocyte. We also counted the numbers of the osteocyte's processes which connected the cytoplasmic processes of the neighboring osteocytes, and then represented the percentage on the numbers of the connecting cytoplasmic processes of an individual osteocyte. The indices of atrophic osteocytes and the connecting cytoplasmic processes of an individual osteocyte were obtained from the same secondary trabecular region used for quantification of the ALPase-positive area.

\section{Statistical analysis}

All statistical analyses were assessed by one-way ANOVA followed by Tukey-Kramer multiple comparisons test, and all values are presented as mean \pm standard deviation. Values of $p<0.05$ were considered significant. 


\section{Results}

\section{Bone volume and osteoclast number after ALN discontinuation}

Compared to control femora (Figs. 2A, I), micro CT imaging and histological sections from specimens obtained after five days of ALN administration showed an increase in bone area (Figs. 2B, J). All specimens obtained after ALN discontinuation maintained the pattern and featured increased metaphyseal trabecular number and area (Figs. 2C-H, K-P). Bone histomorphometry showed that BV/TV (Fig. 2Q) of the specimens after five days of ALN administration and its discontinuation were significantly elevated than control specimens. In contrast, Tb.Sp of ALN-treated specimens significantly decreased compared with that of the control specimens (Fig. 2S). Unlike BV/TV and Tb. Sp. There was no significant changes in Tb.Th and Tb.N (See Figs. 2R, T).

The numbers of TRAPase-positive osteoclasts did not appeared to be increased at 1 to 3 days after ALN discontinuation (Figs. 3A-D), and consistently, there was no statistical difference in the osteoclast numbers in all the experimental periods (Fig. 3E). Thus, osteoclasts did not increase their numbers, without rebounded resorption of once-increased bone volume after the discontinuation of alendronate. At a higher resolution, osteoclasts from control specimens were compared with those from specimens obtained at $0 \mathrm{hr}$ and 3 days after ALN discontinuation (Figs. 4A-F). Immediately after drug discontinuation (at 0hr), many osteoclasts were seen close to the trabecular surfaces (Fig. 4B). However, TEM observation demonstrated several osteoclasts lacked their ruffled borders and clear zones, and did not attach to the bone surfaces (Fig. 4E). At 3 days after the discontinuation, some apoptotic osteoclasts with nuclear condensation could be identified, and several apoptotic osteoclasts with pyknotic nuclei and condensed cell bodies (Figs. 4C, F). Double staining of TUNEL and TRAPase demonstrated apoptotic osteoclasts at 3 days after the discontinuation

(Figs. 4G-I). Statistical analyses showed an increase in the number of TUNEL-positive/TRAPase-reactive osteoclasts after 2days of the ALN discontinuation (Fig. 4J).

\section{Alterations in osteoblasts and osteocytes after ALN discontinuation}

It is well known that ALN inhibits not only osteoclasts, but also affects osteoblastic activity negatively ${ }^{15-18)}$. Therefore, we have examined ALPase-immunoreactivity, a hallmark for osteoblasts, in the region of the secondary metaphyseal trabecules, in which bone remodeling 
takes place in a normal state. Compared with control specimens, ALPase-immunoreactivity seemed lower throughout the period of ALN administration, and appeared to be markedly reduced after the drug discontinuation (Figs. 5A-H). Double staining of TRAPase enzyme activity and ALPase immunoreactivity clearly demonstrated the synchronous reduction of both stains (Figs. 5I-L). Consistent with the histochemical examinations, the statistical analysis revealed a decrease in the indices of ALPase-immunopositive areas not only during the period of ALN administration, but also after its discontinuation (Fig. 5M). Additionally, many osteocytes were atrophic with empty lacunae during and after the ALN treatment (Figs. 6A-D). Under TEM, at 3 days after ALN discontinuation, the number of flattened osteoblasts covering the bone surface were reduced in number, while trabecular osteocytes were shrunken (Fig. 6E). The index of atrophic osteocytes remained elevated at least until the fourth day after ALN discontinuation (Fig. 6F).

\section{ALN-induced osteocyte atrophy}

We assumed that osteocytic atrophy is indirectly linked to ALN-driven osteoblast inhibition, since cytoplasmic processes intimately connect these two cell types in normal conditions. However, while many cuboidal, bone-synthesizing osteoblasts coexisted with atrophic or shrunken osteocytes as early as 36 hrs after a single ALN injection (Figs. 7A, B). When observed under TEM, some osteocytes were shriveled, with amorphous materials in the spaces between the cell body and their lacunar walls, while others were peeling out of the lacunar walls (Figs. 7C-E). The canaliculi of such osteocytes seemed relatively enlarged (Figs. 7F-H). Despite their atrophic profiles, osteocytes tended to keep normal cell organelles and did not seem to be vigorously undergoing apoptosis or nuclear condensation.

We next examined the distribution of rhodamine-phalloidine reactive actin filaments in the osteocytes' cytoplasmic processes by using SIM. Actin filaments in the cytoplasmic processes of ALN-treated osteocytes were disconnected at 36 hrs after the first injection, while control osteocytes extended their cytoplasmic processes and were normally connected to other cells (Figs. 8A, B). The average numbers of cytoplasmic processes of an osteocyte did not change after ALN treatment, but, the index of connecting cytoplasmic processes was significantly decreased at 24, 36 and 48hrs after the single injection (Fig. 8C). Immunoreactivity against chondroitin-4-sulfate, an extracellular substance that normally fills in the osteocytic lacunae and canaliculi ${ }^{32)}$, was markedly reduced after a single ALN injection (Figs. 8 D-I). 


\section{Discussion}

In our murine model of short-term ALN administration, sudden discontinuation of the drug does not seem to induce the resumption of osteoclastic bone resorption, which secures the increased bone volume seen during ALN treatment. However, osteoblastic/osteocytic inhibition appears to be sustained after ALN discontinuation. These findings suggest that osteoblastic and osteocytic activity, more than the restart of osteoclastic bone resorption, may be determinant for cellular and histological events after ALN discontinuation.

While the number of bone-resorbing osteoclasts did not change significantly, osteoclastic apoptosis was increased after ALN administration. When observed under TEM, many multinucleated osteoclasts were not attached to the bone surface nor did they seem to be resorbing the bone matrix, as shown in Fig. 4. Therefore, our findings are consistent with the report by Halasy-Nagy et al., which highlighted that alendronate and risedronate suppress osteoclastic bone resorption by means of other than fostering apoptosis [33]. Notwithstanding, osteoclast precursors would still be able to renew the osteoclast population at the local level even if some osteoclasts went apoptotic, i.e., TUNEL positivity. However, osteoclastic apoptosis was significantly increased at the second day after ALN discontinuation when compared with control specimens. While the precise mechanism behind the phenomenon is hard to explain, we could only consider the possibility that ALPase-immunopositive osteoblasts were inhibited to a point in which their supporting role in osteoclastic differentiation and bone resorption was actually compromised.

One may ponder why osteoblastic activity was reduced during and after ALN administration, as shown in Fig. 5. In vivo animal studies have shown that bisphosphonate administration negatively affects mineral apposition rate, which suggests reduced osteoblast activity [15-18]. This may be due to hindrances in the "coupling phenomenon", the partnership with osteoclasts that seems to be necessary for osteoblastic activity during bone remodeling [12-14]. High-dose bisphosphonate administration disrupts the coupling between osteoclastic bone resorption and osteoblastic bone formation [34], and several in vivo studies have shown the effects of such disruption of cell coupling on osteoblasts [17, 35]. Therefore, we inferred that disrupted cell coupling was the reason why osteoblastic activity was reduced during and after ALN administration. 
Then, why would not the number of ALPase-positive osteoblasts immediately increase after ALN discontinuation? Fuchs et al, in agreement with our findings here, have reported that ALN had a more persistent suppressive effect on bone turnover than did risedronate after treatment was withdrawn [36]. Since osteoblasts extend their cytoplasmic processes and connect with neighboring osteoblasts and osteocytes embedded in bone matrix, we considered the hypothesis that more time is needed for osteoblasts to reorganize their functional syncytium.

On the other hand, the effect of ALN on osteocytes is also worth mentioning. Low ALN doses seem to suppress osteocytic apoptosis by affecting the permeability of channels formed by connexin 43 [37-39]. Since we used young adult mice in the present study, we did not see high numbers of apoptotic osteocytes in a normal state, and it is therefore hard to assess whether ALN has any anti-apoptotic effects on osteocytes. However, at the electron microscope level, osteocytes did show atrophic features - the shrunken cell bodies peeling out of their lacuna walls, as shown in Fig. 6. Since cell organelles seemed unaltered in those osteocytes, we cannot agree with the notion that ALN fosters osteocytic apoptosis. Interestingly though, the disconnection among cytoplasmic processes shown by SIM, along with the reduced chondroitin-4-sulfate immunoreactivity and the amorphous materials observed under TEM within the osteocytic lacunae may reflect an altered state of the osteocytic lacunar-canalicular system, which may affect the functionality of the osteocytic syncytium as a whole.

An interesting finding of this study was the fact that atrophic osteocytes showed atrophy coexisted with plump mature osteoblasts, as shown in Fig. 7. This suggests a rather direct effect of ALN upon osteocytes. We had thought, at first, that ALN affects osteoblastic activity negatively by disrupting cell coupling with osteoclasts and subsequently forces osteocytes into atrophy. However, there seems to be a direct effect of ALN on osteocytes. While it is hard to understand how ALN molecules reach to the osteocytes, these cells may incorporate a small amount of the drug by pinocytosis. Coxon et al have demonstrated that calvarial osteoblasts and MCF-7 tumor cells internalized a small amount of ALN and risedronate coated on dentin slices [40]. It is feasible that osteocytes incorporate ALN molecules and then become atrophic, which would make full osteoblastic recovery after ALN discontinuation even more challenging given the osteocyte's position as one of the regulators of osteoblastic activity. 


\section{Conclusion}

Our findings based on a murine model of short-term ALN administration followed by its discontinuation suggest that ALN may have a persistent suppressive effect on osteoblastic and osteocytic activities. This may cast a shadow over recommendations regarding drug holidays for patients on ALN, since the drug might have somewhat lasting effects on bone turnover. 


\section{Acknowledgments}

This study was partially supported by the Grants-in Aid for Scientific Research (Amizuka N, Hasegawa T, Tsuboi K) and Promoting International Joint Research (Bilateral Collaborations) of JSPS and NSFC (Amizuka N, Li M). 


\section{References}

1. Amin D, Cornell SA, Gustafson SK, Needle SJ, Ullrich JW, Bilder GE, Perrone MH (1992) Bisphosphonates used for the treatment of bone disorders inhibit squalene synthase and cholesterol biosynthesis. J Lipid Res 33:1657-1663

2. Rogers MJ (2003) New insights into the molecular mechanisms of action of bisphosphonates. Curr Pharm Des 9:2643-2626

3. Luckman SP, Hughes DE, Coxon FP, Graham R, Russell G Rogers MJ (1998) Nitrogen-containing bisphosphonates inhibit the mevalonate pathway and prevent post-translational prenylation of GTP-binding proteins, including Ras. J Bone Miner Res13:581-589

4. Palokangs H, Mulari M, Vaananen HK (1997) Endocytic pathway from the basal plasma membrane to the ruffled border membrane in bone resorbing osteoclasts. J Cell Sci 110:1767-1860

5. Abu-Amer Y, Teitelbaum SL, Chappel JC, Schlesinger P, Ross FP (1999) Expression and regulation of RAB3 proteins in osteoclasts and their precursors. J Bone Miner Res $11: 1855-1860$

6. Alakangas A, Selander K, Mulari M, Halleen J, Lehenkari P, Mönkkönen J, Salo J, Väänänen K (2002) Alendronate disturbs vesicular trafficking in osteoclasts. Calcif Tissue Int 70:40-47

7. Sato M, Grasser W, Endo N, Akins R, Simmons H, Thompson DD, Golub E, Rodan GA (1991) Bisphosphonate action. Alendronate localization in rat bone and effects on osteoclast ultrastructure. J Clin Invest 88:2095-2105

8. Frost HM (1969) Tetracycline-based histological analysis of bone remodeling. Calcif Tissue Res 3:211-237

9. Hattner R, Epker BN, Frost HM (1965) Suggested sequential mode of control of changes in cell behaviour in adult bone remodelling. Nature 206:489-490 
10. Frost HM (1964) Dynamics of bone remodeling. In Bone Biodynamics. H.M.Frost ed. Boston: Little Brown, p.315-333

11. Baron R, Vignery A, Horowits M (1983) Lymphocytes, macrophages and the regulation of bone remodeling. Bone and Mineral Research 2:175-243

12. Nishino I, Amizuka N, Ozawa H (2001) Histochemical examination of osteoblastic activity in op/op mice with or without injection of recombinant M-CSF. J Bone Miner Metab 19:267-276

13. Sakagami N, Amizuka N, Li M, Takeuchi K, Hoshino M, Nakamura M, Nozawa-Inoue K, Udagawa N, Maeda T (2005) Reduced osteoblastic population and defective mineralization in osteopetrotic (op/op) mice. Micron 36:688-695

14. Luiz de Freitas PH, Li M, Ninomiya T, Nakamura M, Ubaidus S, Oda K, Udagawa N, Maeda T, Takagi R, Amizuka N (2009) Intermittent PTH administration stimulates pre-osteoblastic proliferation without leading to enhanced bone formation in osteoclast-less c-fos(-/-) mice. J Bone Miner Res 24:1586-1597

15. Bikle DD, Morey-Holton ER, Doty SB, Currier PA, Tanner SJ, Halloran BP (1994) Alendronate increases skeletal mass of growing rats during unloading by inhibiting resorption of calcified cartilage. J Bone Miner Res 9:1777-1787

16. Iwata K, Li J, Follet H, Phipps RJ, Burr DB (2006) Bisphosphonates suppress periosteal osteoblast activity independently of resorption in rat femur and tibia. Bone 39: 1053-1058

17. Nakamura M, Udagawa N, Matsuura S, Mogi M, Nakamura H, Horiuchi H, Saito N, Hiraoka BY, Kobayashi Y, Takaoka K, Ozawa H, Miyazawa H, Takahashi N (2003) Osteoprotegerin regulates bone formation through a coupling mechanism with bone resorption. Endocrinology 144:5441-5449

18. Mashiba T, Turner CH, Hirano T, Forwood MR, Johnston CC, Burr DB (2001) Effects of suppressed bone turnover by bisphosphonates on microdamage accumulation and biomechanical properties in clinically relevant skeletal sites in beagles. Bone 28:524-531

19. Ubaidus S, Li M, Sultana S, de Freitas PH, Oda K, Maeda T, Takagi R, Amizuka N (2009) FGF23 is mainly synthesized by osteocytes in the regularly distributed osteocytic lacunar 
canalicular system established after physiological bone remodeling. J Electron Microsc 58:381-392

20. Amizuka N, Hasegawa T, Oda K, Freitas PHL, Hoshi K, Li M, Ozawa H (2012) Histology of epiphyseal cartilage calcification and endochondral ossification. Front Biosci 4: 2085-2100

21. Sasaki M, Hongo H, Hasegawa T, Suzuki R, Liu Z, Freitas PHL, Yamada T, Oda K, Yamamoto T, Li M, Totsuka Y, Amizuka N (2012) Morphological aspects on osteocytic function on bone mineralization. Oral Science International 9:1-8

22. Doty SB (1981) Morphological evidence of gap junctions between bone cells. Calcif Tissue Int 33:509-512

23. Stains JP, Civitelli R (2005) Gap junctions in skeletal development and function. Biochem Biophys Acta 1719:69-81

24. Stains JP, Watkins MP, Grimston SK, Hebert C, Civitelli R (2014) Molecular mechanisms of osteoblast/osteocyte regulation by connexin43. Calcif Tissue Int 94:55-67

25. Marx RE. Pamidronate (Aredia) and zoledronate (Zometa) induced avascular necrosis of the jaws: a growing epidemic (2003) J Oral Maxillofac Surg 61:1115-1117

26. Marx RE, Cillo JE Jr, Ulloa JJ (2007) Oral bisphosphonate-induced osteonecrosis: risk factors, prediction of risk using serum CTX testing, prevention, and treatment. J Oral Maxillofac Surg 65:2397-2410

27. Hasegawa T, Ri S, Umeda M, Komatsubara H, Kobayashi M, Shigeta T, Yoshitomi I, Ikeda H, Shibuya Y, Asahina I, Komori T (2013) The observational study of delayed wound healing after tooth extraction in patients receiving oral bisphosphonate therapy. $\mathrm{J}$ Craniomaxillofac Surg 41:558-563

28. Amizuka N, Kwan M, Goltzman D, Ozawa H, White JH (1999) Vitamin D3 differentially regulates parathyroid hormone/parathyroid hormone-related peptide receptor expression in bone and cartilage. J. Clin. Invest 103:373-381

29. Sasaki M, Hasegawa T, Yamada T, Hongo H, de Freitas PH, Suzuki R, Yamamoto T, Tabata C, Toyosawa S, Yamamoto T, Oda K, Li M, Inoue N, Amizuka N (2013) Altered distribution of bone matrix proteins and defective bone mineralization in klotho-deficient 
mice. Bone 57:206-219

30. Oda K, Amaya Y, Fukushi-Irie M, Kinameri Y, Ohsuye K, Kubota I, Fujimura S, Kobayashi J (1999) A general method for rapid purification of soluble versions of glycosylphosphatidylinositol-anchored proteins expressed in insect cells: An application for human tissue-nonspecific alkaline phosphatase. J. Biochem. 126:694-699

31. Amizuka N, Takahashi N, Udagawa N, Suda T, Ozawa H (1997) An ultrastructural study of cell-cell contact between mouse spleen cells and calvaria-derived osteoblastic cells in a co-culture system for osteoclast formation. Acta Histochem. Cytochem 30:351-362

32. Takagi M,Ono Y, Maeno M, Miyashita K, Omiya K (1997) Immunohistochemical and biochemical characterization of sulphated proteoglycans in embryonic chick bone. J Nihon Univ Sch Dent 39:156-163

33. Halasy-Nagy JM, Rodan GA, Reszka AA (2001) Inhibition of bone resorption by alendronate and risedronate does not require osteoclast apoptosis. Bone 29:553-539

34. Sama AA, Khan SN, Myers ER, Huang RC, Cammisa FP Jr, Sandhu HS, Lane JM (2004) High-dose alendronate uncouples osteoclast and osteoblast function: a study in a rat spine pseudarthrosis model. Clin Orthop Relat Res 425:135-142

35. Shimizu E, Tamasi J, Partridge NC (2012) Alendronate affects osteoblast functions by crosstalk through EphrinB1-EphB. J Dent Res 91:268-274

36. Fuchs RK, Phipps RJ, Burr DB (2008) Recovery of trabecular and cortical bone turnover after discontinuation of risedronate and alendronate therapy in ovariectomized rats. $\mathrm{J}$ Bone Miner Res 23:1689-1697

37. Plotkin LI, Weinstein RS, Parfitt AM, Roberson PK, Manolagas SC, Bellido T (1999) Prevention of osteocyte and osteoblast apoptosis by bisphosphonates and calcitonin. J Clin Invest 104:1363-1374

38. Plotkin LI, Manolagas SC, Bellido T (2002) Transduction of cell survival signals by connexin-43 hemichannels. J Biol Chem 277:8648-8657

39. Plotkin LI, Aguirre JI, Kousteni S, Manolagas SC, Bellido T (2005) Bisphosphonates and estrogens inhibit osteocyte apoptosis via distinct molecular mechanisms downstream of 
extracellular signal-regulated kinase activation. J Biol Chem 280:7317-7325

40. Coxon FP, Thompson K, Roelofs AJ, Ebetino FH, Rogers MJ (2008) Visualizing mineral binding and uptake of bisphosphonate by osteoclasts and non-resorbing cells. Bone 42:848-860

\section{Figure Legends}

\section{Figure 1}

\section{Experimental schedule}

Six weeks-old female ICR mice are received daily subcutaneous injections of $1 \mathrm{mg} / \mathrm{kg}$ of ALN (black arrows) for 10 days. Mice are fixed at 5 days after the first injection and the others are fixed immediately ( 0 hr), 12 hrs, 1, 2, 3 and 4 days after the last injection (white arrows).

\section{Figure 2}

Micro CT images, histology and bone histomorphometry of static parameters of murine femora treated with ALN

Panels A-H are sagittal images of femoral metaphyses obtained by micro CT, while panels I-P are H-E sections of femoral metaphyses of the control mice (without ALN; A, I), at 5 days after the first ALN injection (B, J) and $0 \mathrm{hr}(\mathbf{C}, \mathbf{K}), 12 \mathrm{hrs}(\mathbf{D}, \mathbf{L}), 1$ day (E, M), 2 days (F, N), 3 days $(\mathbf{G}, \mathbf{O})$ and 4 days $(\mathbf{H}, \mathbf{P})$ after ALN discontinuation. ALN-treated specimens $(\mathbf{B}-\mathbf{H}$, $\mathbf{J}-\mathbf{P})$ show the increased metaphyseal bone compared to those of the control femora (A, I), featuring the similar histology between micro CT mages (B-H) and histological sections (J-P). Panels Q-T are histomorphometric analyses of BV/TV (Q), Tb.Th (R), Tb.Sp (S) and Tb.N (T). The index of BV/TV after ALN administration becomes significantly higher than that of control specimen, and dose not decrease even after ALN discontinuation (Q). In contrast, Tb.Sp significantly decreases after the discontinuation (S). There is no significant difference in Tb.Th (R) and Tb.N (T). ${ }^{* *} p<0.01$ and ${ }^{*} p<0.05$ versus control groups, using One-way ANOVA, Tukey-Kramer test (See M\&M)

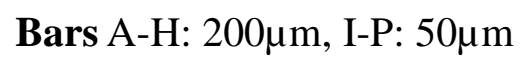

\section{Figure 3}

\section{Distribution of TRAPase-positive osteoclasts after the ALN discontinuation}

TRAPase enzyme histochemistry demonstrate the distribution of osteoclasts (red, white arrows) in the metaphyses of ALN-treated mice of the control (without ALN, A), 1 day (B), 2 days (C), 3 days (D) of its discontinuation. Statistical analysis on the numbers of TRAPase-positive osteoclasts shows no significant difference in all the experimental periods 
(E).

Bars A-D: 70 $\mu \mathrm{m}$

\section{Figure 4}

Light microscopic (A-C) and TEM (D-F) observations on apoptotic osteoclasts, and double staining of TRAPase/TUNEL (G-I) after the ALN discontinuation

Semithin sections demonstrate many osteoclasts (oc) on the trabecular surface of the control specimens (without ALN; A) and those at $0 \mathrm{~h}$ after the ALN discontinuation (B). However, TEM observation demonstrates well-developed ruffled borders (RB) of the control osteoclasts (D), while, at Ohr after the discontinuation, several osteoclasts without ruffled borders (white arrows) and clear zones, which do not attach to the bone surfaces (E). After three days of the ALN discontinuation, many apoptotic osteoclasts (white arrows) are seen in the metaphyses (C). When observing under TEM, spherical osteoclasts (asterisks) with pyknotic nuclei can be seen (F). Panel G-I show double staining of TRAPase-reactive/TUNEL-positive osteoclasts. Please notice double positive osteoclasts for TUNEL (brown) and TRAPase (red) at 3 days after the discontinuation (an inset of $\mathbf{I}$ ). Panel $\mathbf{J}$ is the statistical analysis on the index of TRAPase-reactive/TUNEL-positive osteoclasts, featuring a significant increase of apoptotic osteoclasts between the control and the experimental specimens at 2, 3 and 4 days after ALN discontinuation. ${ }^{* *} p<0.01$ and ${ }^{*} p<0.05$ versus control groups, using One-way ANOVA, Tukey-Kramer test. ob: osteoblast

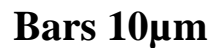

\section{Figure 5}

ALPase immunohistochemistry and double detection of TRAPase and ALPase in the metaphyses

Panels A-H show ALPase immunohistochemistry of the control specimen (without ALN, A), and those at 5 days after the first injection (B), and 0 hr (C), 12 hrs (D), 1 day (E), 2 days (F), 3 days (G) and 4 days $(\mathbf{H})$ after the drug withdrawn of ALN medication. Note that ALP-immunoreactivity is chronologically reduced in the region of the secondary trabecules. Panels I-L are double staining of TRAPase reactive osteoclasts (white arrows) and ALPase immunopositive osteoblasts (black arrows), clearly demonstrating the synchronous reduction of both stains. Statistical analysis reveals a significantly decreased index of ALPase-immunopositive areas not only during the ALN administration but also after its 
discontinuation (M). $\quad * * p<0.01$ versus control and after 0h groups, using One-way ANOVA, Tukey-Kramer test.

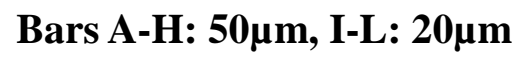

Figure 6

Light microscopic and TEM images of osteocytes after the withdrawn of ALN medication

Compared with osteocytes (ocy) of the control specimens (A), shrunken osteocytes with showing opening areas in their lacunae can be seen at $0 \mathrm{hr}$ (B), 1 day (C) and 4 days (D) after the ALN treatment. Under TEM observations, there are a few flattened osteoblasts (a black arrow) covering bone surface, and degenerated osteocytes (ocy) in the trabecule at 3 days after the discontinuation (E). Statistical analysis reveals that the highly-elevated index of osteocytic atrophy remains at least until the 4 days after ALN discontinuation (F). $\quad{ }^{* *} p<0.01$ versus control groups, using One-way ANOVA, Tukey-Kramer test.

\section{Bars, 10 Hm}

\section{Figure 7}

\section{Ultrastructural alterations in osteocytes at $36 \mathrm{hs}$ after a single ALN injection}

Semithin sections demonstrate that many cuboidal, bone-synthesizing osteoblasts coexist with atrophic or shrunken osteocytes (ocy, A, B). Panel $\mathbf{B}$ is a higher magnification of a boxed area in A. Under TEM observation, a control osteocyte shows a round shape and the matched configuration with the lacuna (C). However, in the ALN-treated sections, the osteocyte seems atrophic, being localized with amorphous materials (an asterisk) in their lacuna (D). Another osteocyte is shown to be peeled out of the wall of its lacuna (E). The canaliculi (arrows) of such osteocytes tend to be relatively enlarged in diameters compared to control osteocytes (F-G).

Bars: A, B: $10 \mu \mathrm{m}, \mathrm{C}-\mathrm{E}: 2 \mu \mathrm{m}, \mathrm{F}-\mathrm{H}: 1 \mu \mathrm{m}$

\section{Figure 8}

Distribution of actin filaments in the osteocytes' cytoplasmic processes and Chondroitin-4-sulfate immunoreactivity

Under SIM observation, control osteocytes (ocy) well-extend their cytoplasmic processes (red, actin filaments) to connect between neighboring osteocytes (A), while cytoplasmic processes 
of ALN-treated osteocytes are disconnected at 36 hrs after the single injection (arrows, B). The average numbers of cytoplasmic processes of an individual osteocyte do not change, but a percentage of connecting cytoplasmic processes of an osteocyte are shown to be decreased after the single injection of ALN (C). ${ }^{* *} p<0.05$ versus control groups, using One-way ANOVA, Tukey-Kramer test. Panels D-I represent immunolocalization of chondroitin-4-sulfate in control osteocyte (D) and ALN-treated osteocytes at 3hrs (E), 9hrs (F), 24hrs (G), 36hrs (H) and 48 hrs (I) after the single injection. Notice gradually-reduced immunoreactivity of chondroitin-4-sulfate (arrows).

\section{Bars: $5 \mu \mathrm{m}$}




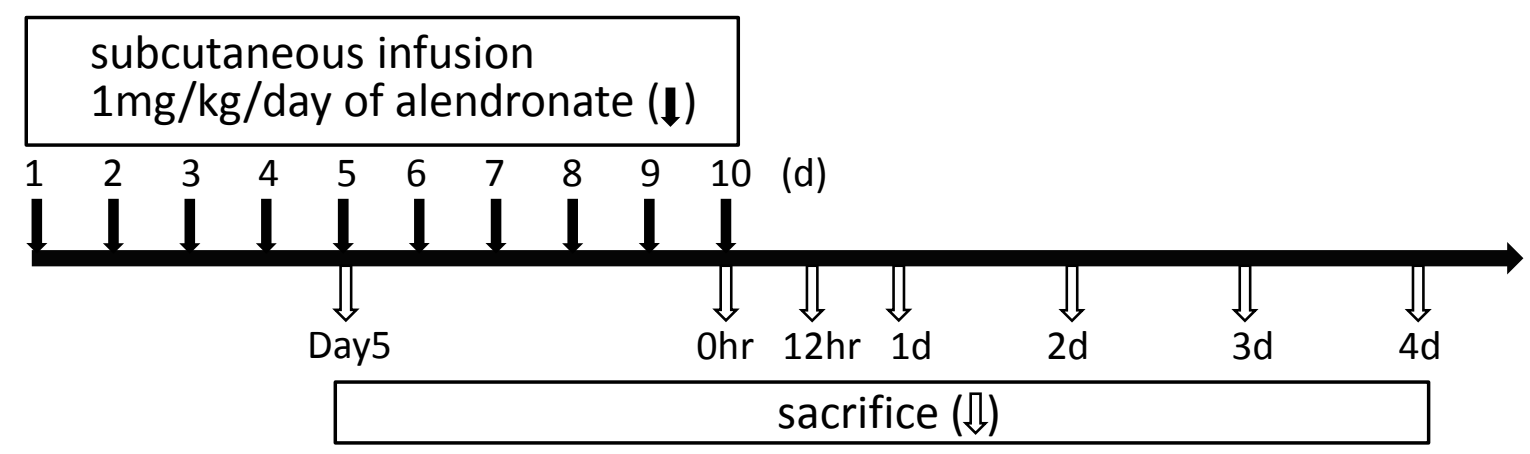

Figure 1 

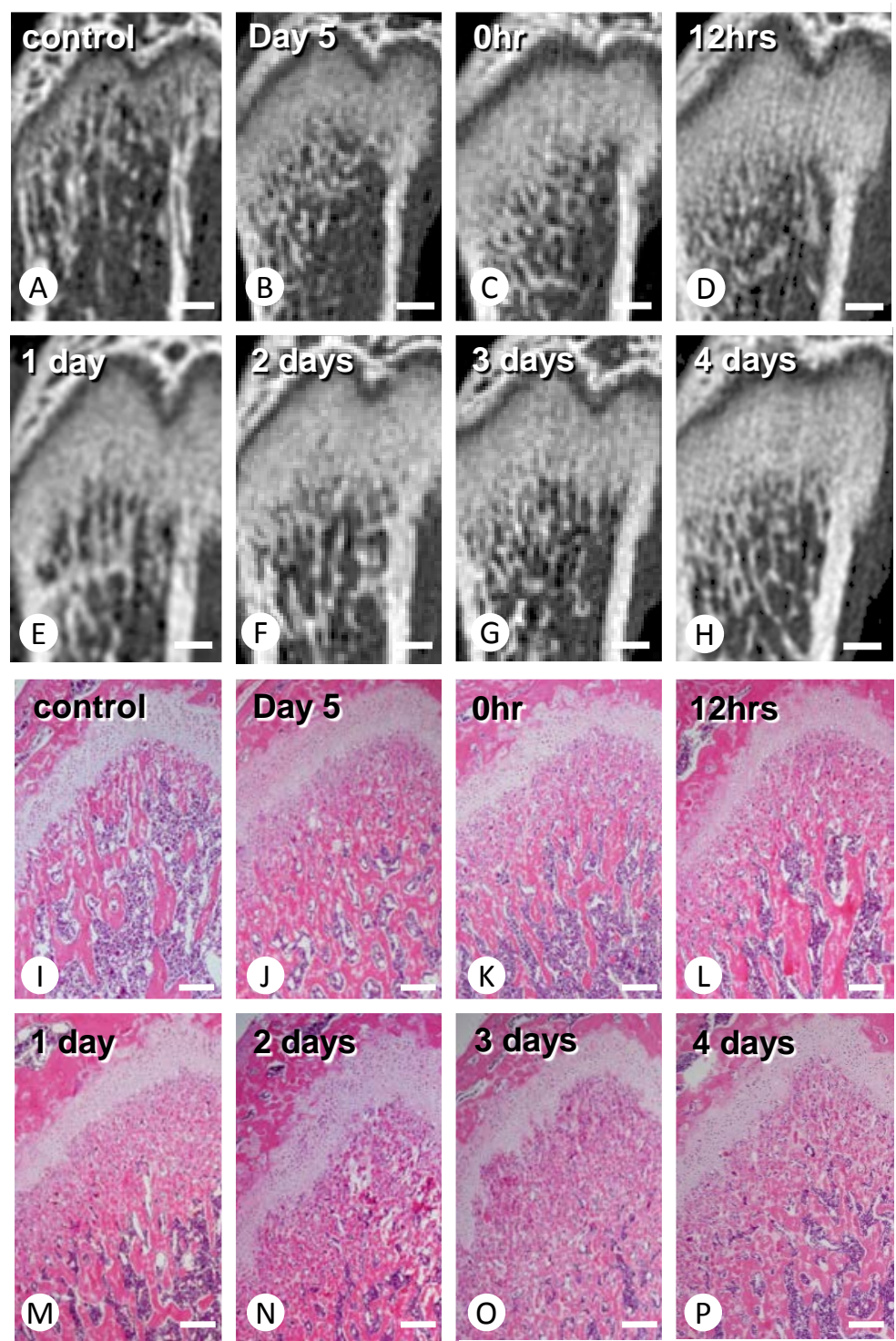

BVITV
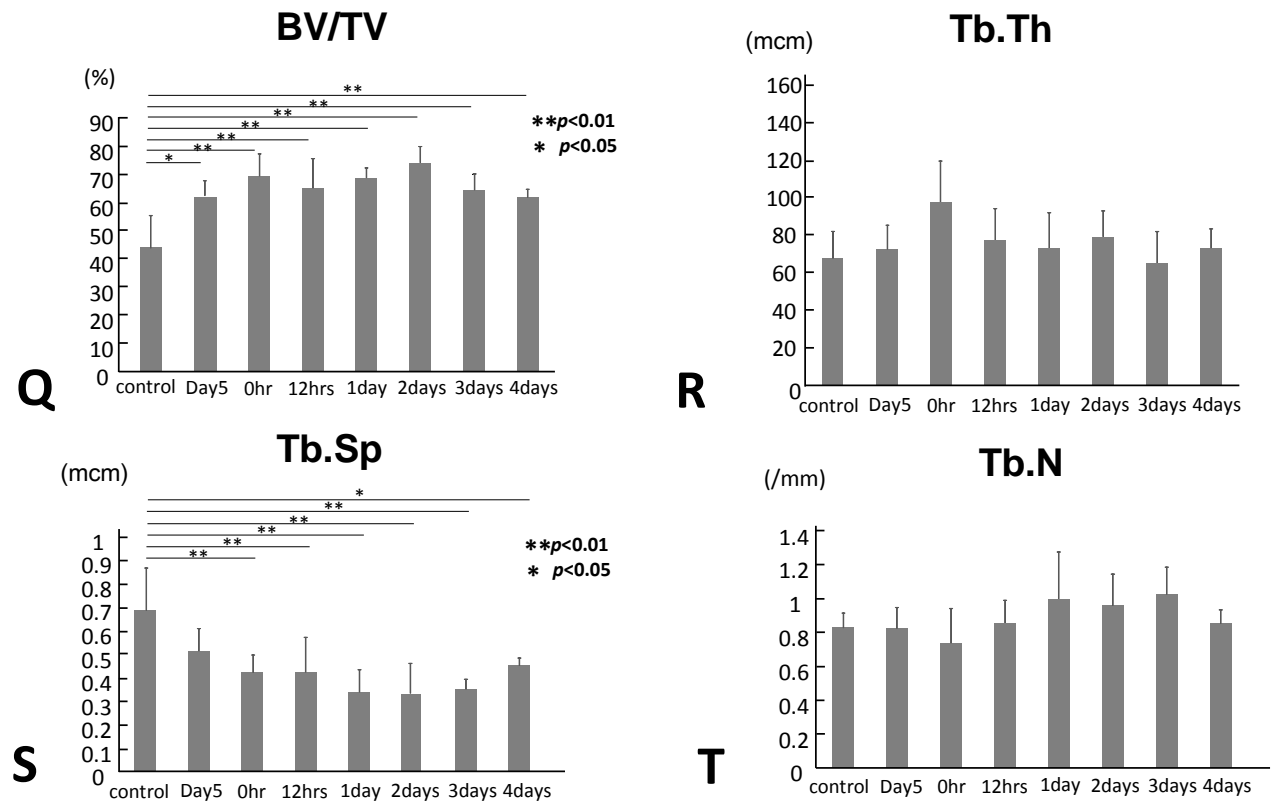

Figure 2 

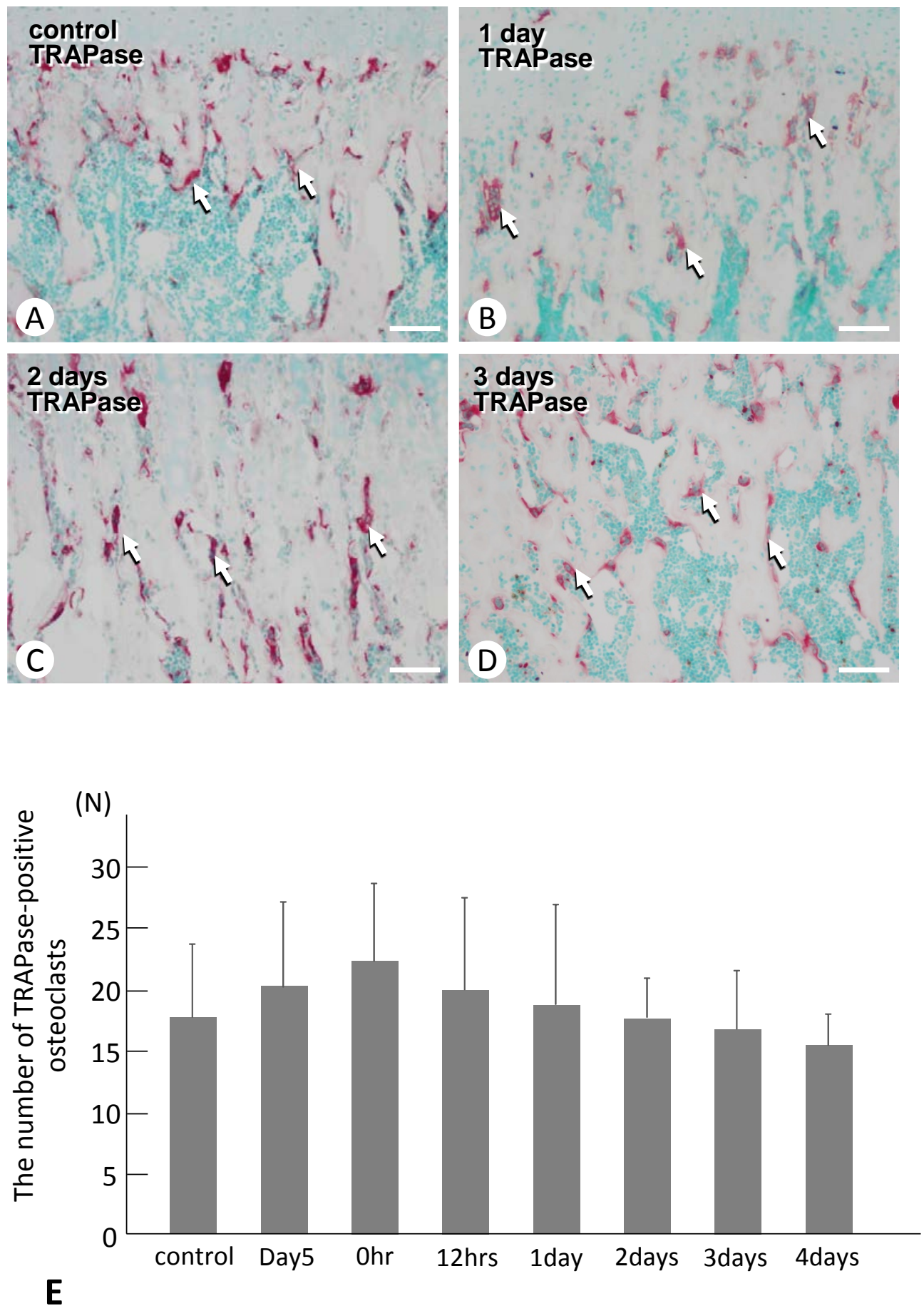

Figure 3 

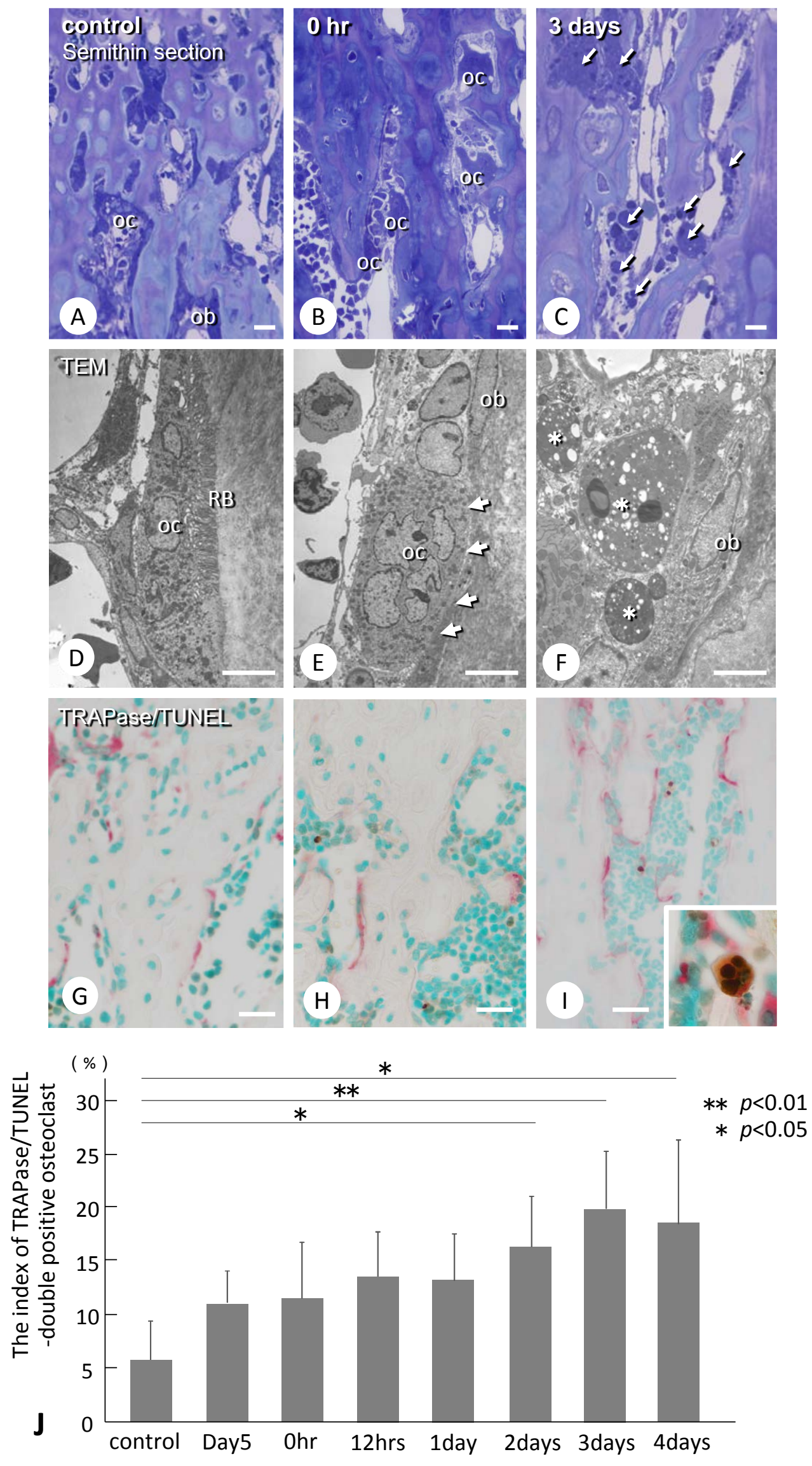

Figure 4 

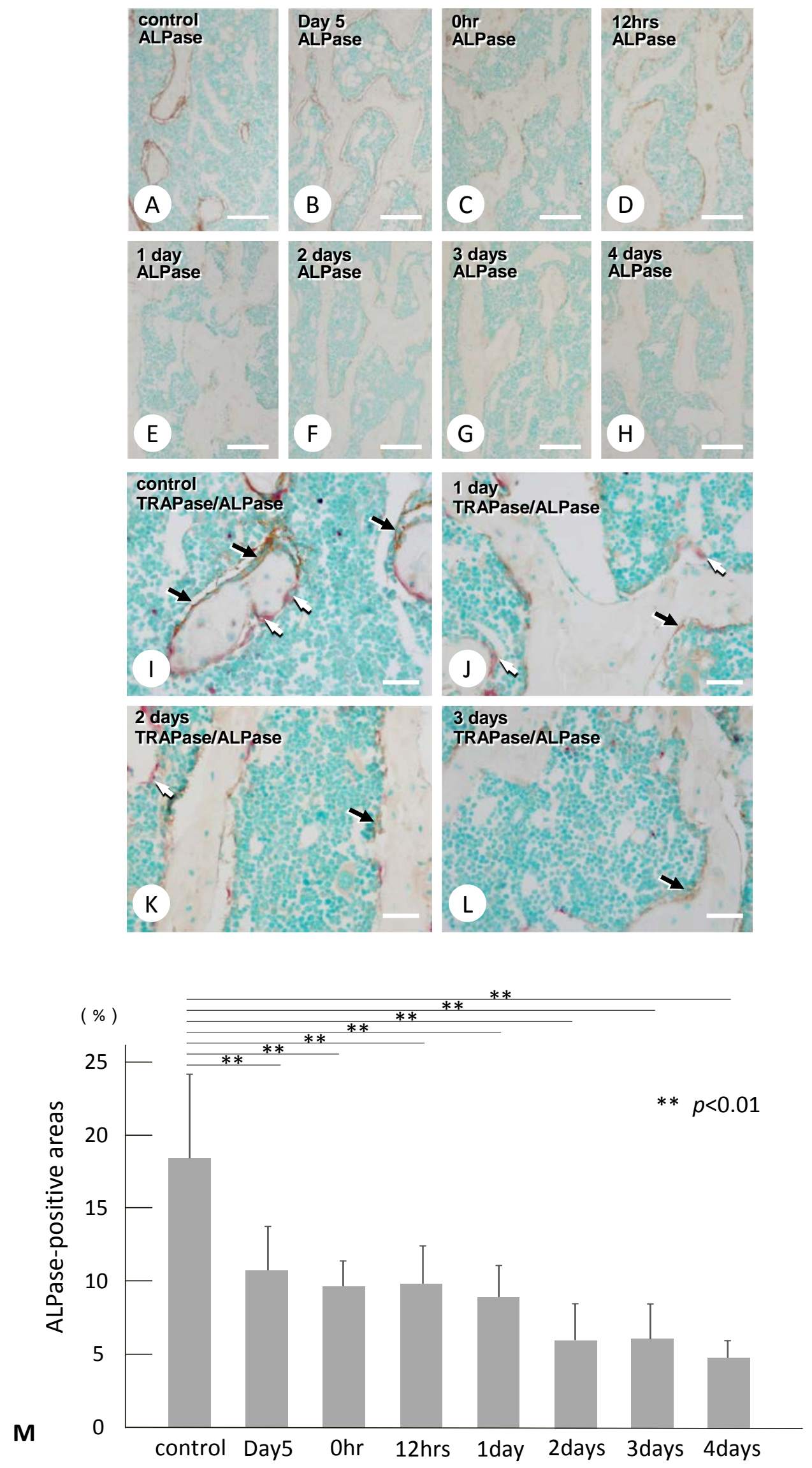

Figure 5 

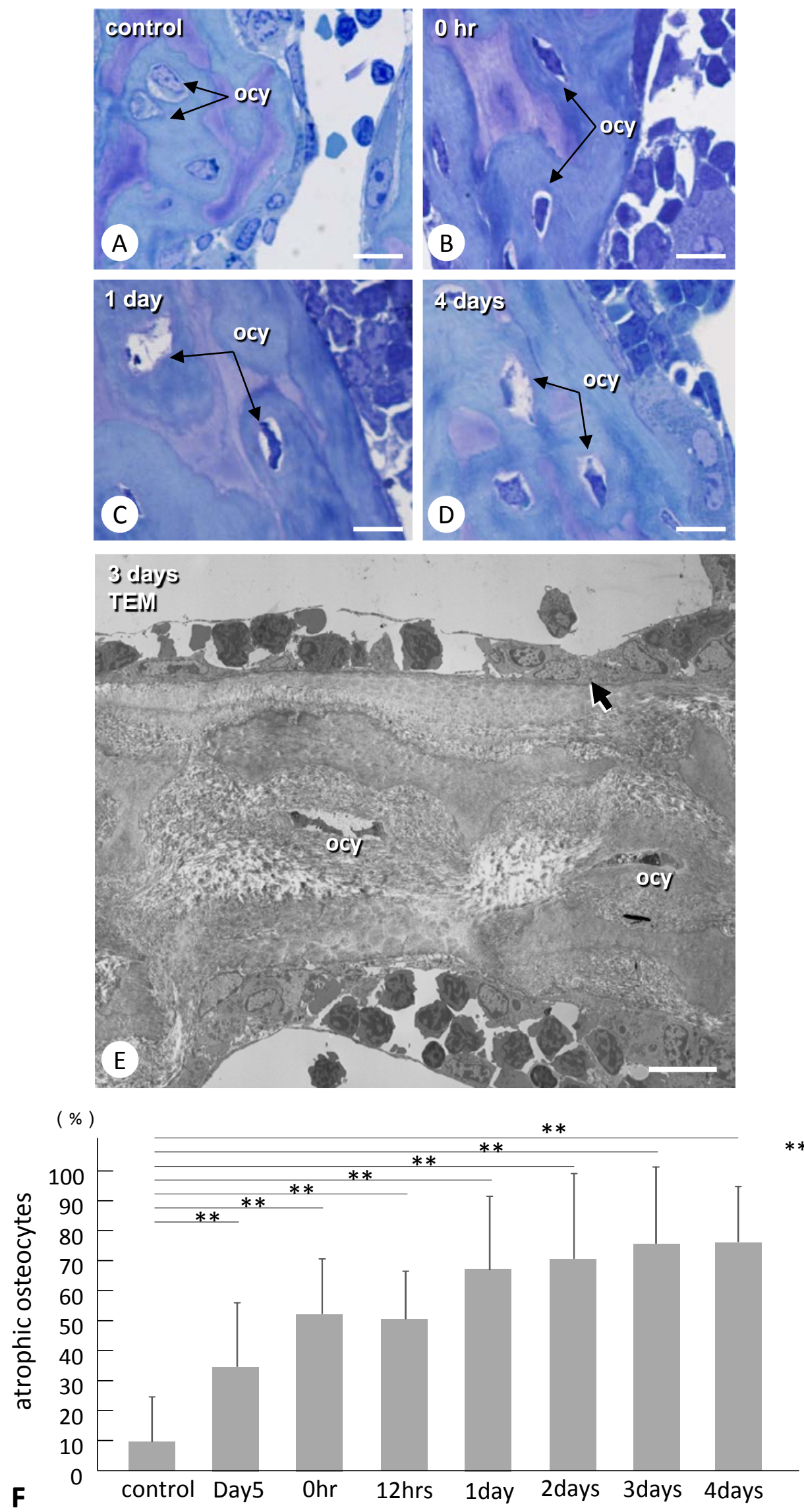

Figure 6 


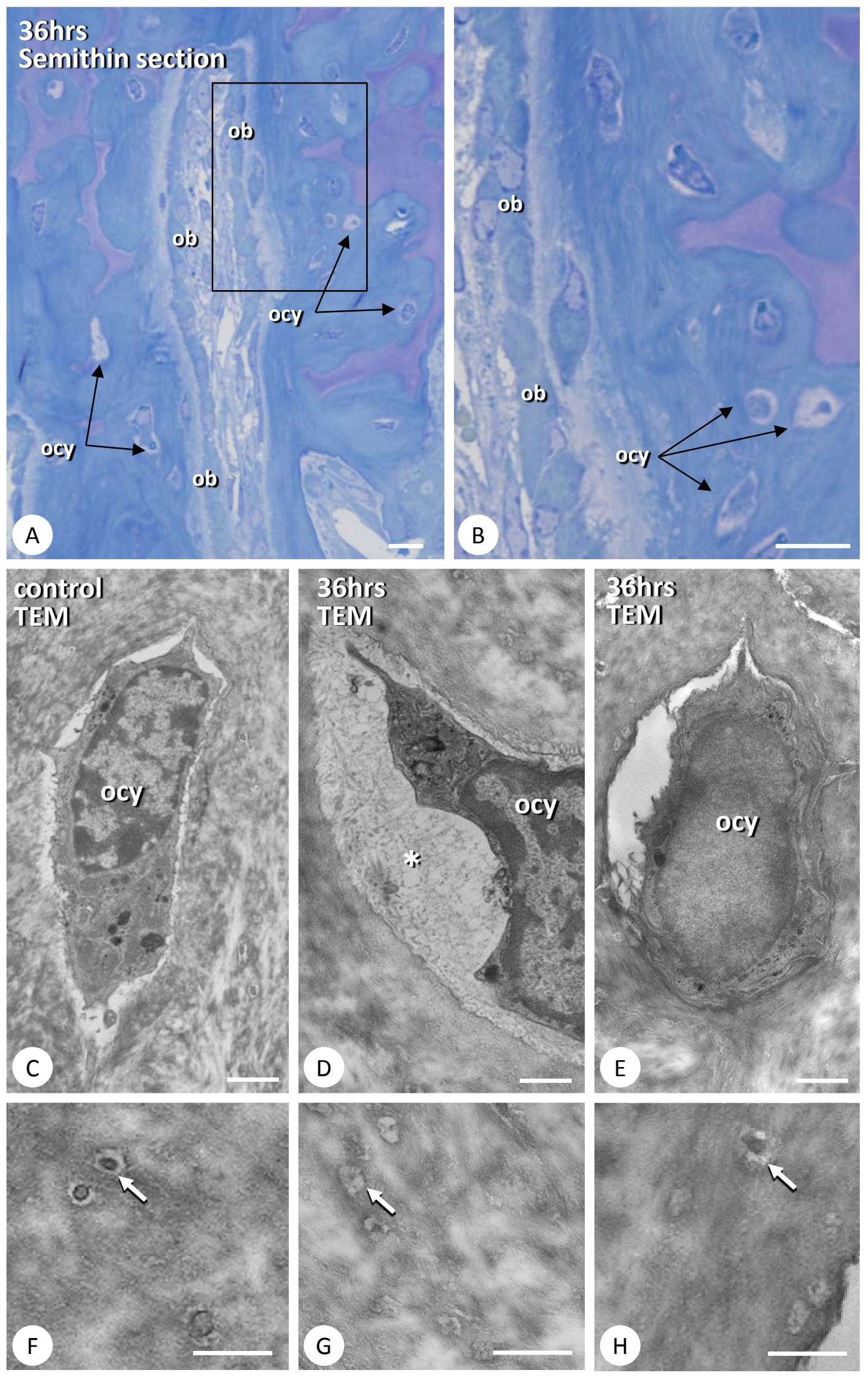

Figure 7 


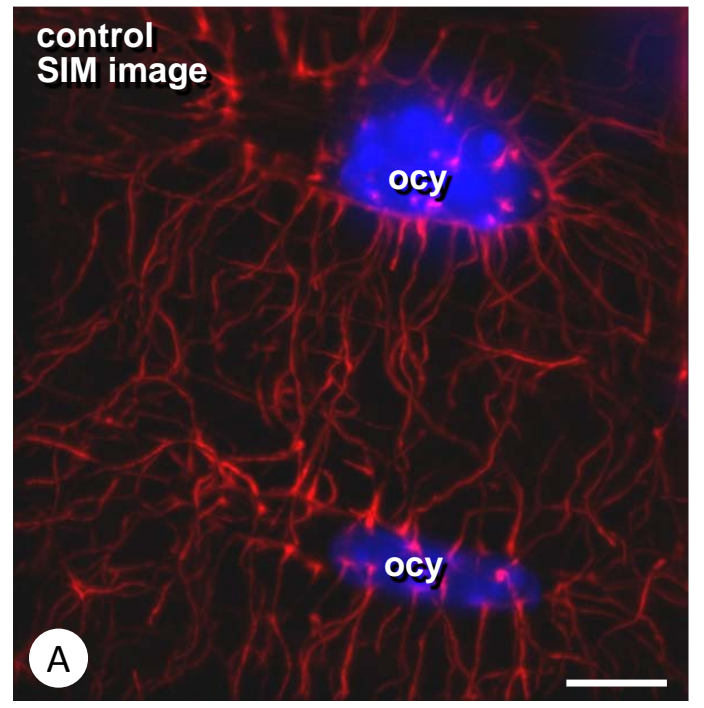

\title{
36hrs \\ SIM image
}

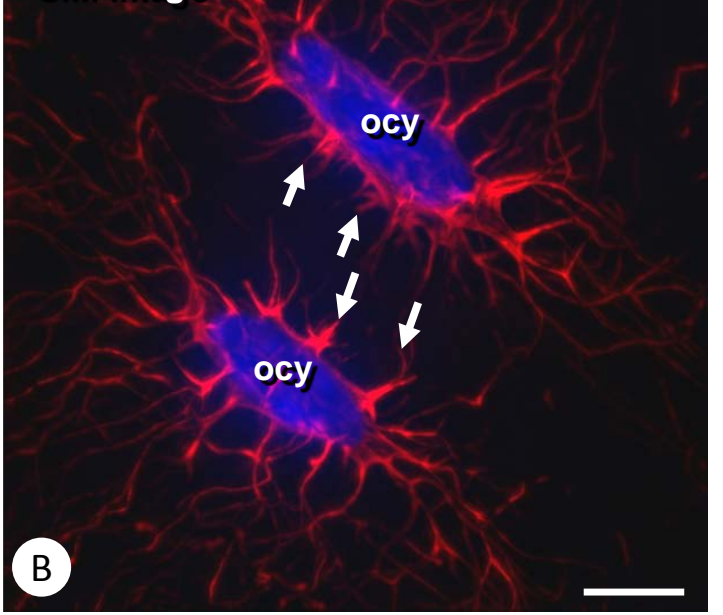

\begin{tabular}{lllllll} 
control & $3 \mathrm{hrs}$ & $9 \mathrm{hrs}$ & $24 \mathrm{hrs}$ & $36 \mathrm{hrs}$ & $48 \mathrm{hrs}$ \\
\hline
\end{tabular}

The average numbers of cytoplasmic processes per one osteocyte

\section{$18.9 \pm 2.55$}

\author{
$18.7 \pm 4.99$
}

$18.0 \pm 2.02$

$15.0 \pm 1.60 \quad 16.0 \pm 3.22 \quad 19.7 \pm 3.76$

The percentage of connecting cytoplasmic processes per one osteocyte

\begin{tabular}{|c|c|c|c|c|c|}
\hline $95.8 \pm 6.99$ & $91.0 \pm 5.89$ & $84.2 \pm 4.18$ & $77.1 \pm 4.33$ & $\begin{array}{r}* * \\
55.4 \pm 13.5\end{array}$ & $61.8 \pm 3.94$ \\
\hline
\end{tabular}
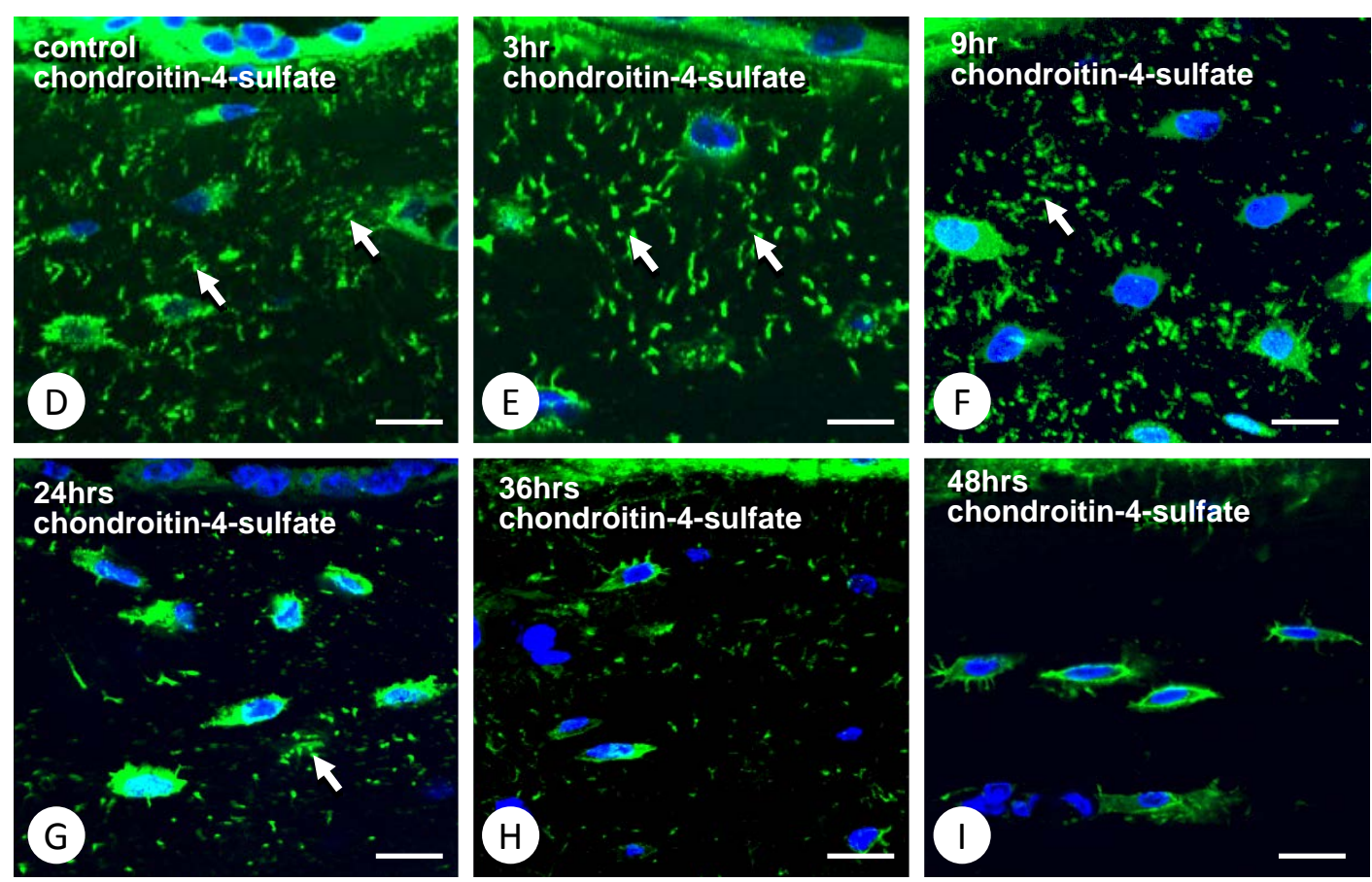

Figure 8 